\title{
3D quantum Hall effects and nonlinear Hall effect
}

\author{
Shuai Li $\mathbb{I D}^{1,2,3}$, C. M. Wang ${ }^{2,3,4}$, Z. Z. Du ${ }^{2,3}$, Fang Qin $\mathbb{D}^{2,3}$, Hai-Zhou Lu ${ }^{2,3 凶}$ and X. C. Xie ${ }^{5,6,7}$
}

The classical and quantum Hall effects are important subjects in condensed matter physics. The emergent 3D quantum Hall effects and nonlinear Hall effect have attracted considerable interest recently, with the former elevating the quantum Hall effect to a higher dimension and the latter extending the Hall effect to higher-order responses. In this perspective, we briefly introduce these two new members of the Hall family and discuss the open questions and future research directions.

npj Quantum Materials (2021)6:96; https://doi.org/10.1038/s41535-021-00399-2

\section{INTRODUCTION}

The Hall effects have long been research focus in condensed matter physics ${ }^{1-3}$. In particular, the quantum Hall effect ${ }^{4,5}$, which manifests as the quantized Hall resistance and zero longitudinal resistance of the two-dimensional electron gas in a strong magnetic field, is one of the greatest discoveries in physics. Thus far, there have been four main generalizations of the quantum Hall effect: fractionalization, no magnetic field, higher dimension, and nonlinearity. The first generalization, the fractional quantum Hall effect, was the subject of the 1998 Nobel Prize in Physics ${ }^{6,7}$. The study of the quantum Hall effect in the absence of a magnetic field has led to the discoveries of several topological states of matter ${ }^{8-17}$. Recently, rapid progress has been made on the latter two generalizations, that is, the 3D quantum Hall effects and the nonlinear Hall effect. In this perspective, we introduce these two new members of the Hall family, focusing on both experimental and theoretical aspects, and discuss the open questions and future directions.

\section{D QUANTUM HALL EFFECTS}

Researchers have been attempting to realize the quantum Hall effect in 3D systems over 30 years ${ }^{18-31}$. Recently, the quantizations of Hall conductance and Hall conductivity have been observed in $3 \mathrm{D}$ devices of $\mathrm{Cd}_{3} \mathrm{As}_{2}{ }^{32-37}$ and $\mathrm{ZrTe}_{5}{ }^{38}$, respectively. The former is based on topologically protected Fermi arcs in topological semimetals, and the latter is believed to arise from the chargedensity wave (CDW) of the Landau bands.

In topological semimetals, the conduction and valence bands touch at the Weyl points. The Fermi arcs are the Fermi surface of the topologically protected surface states of the topological semimetals (Fig. 1a). At a single surface, the Fermi-arc surface states cannot form a complete 2D electron gas to support the cyclotron motion of electrons, which rules out the Landau levels and quantum Hall effect. It has been proposed that the 2D Fermiarc surface states from different surfaces can be connected at the Weyl points ${ }^{39-41}$ to form a complete 2D electron gas to support the $3 \mathrm{D}$ quantum Hall effect ${ }^{32,33}$ (Fig. $1 \mathrm{~b}$ ). In real space, driven by a perpendicular magnetic field, an electron performs half of a cyclotron motion on the top surface and then tunnels to the bottom surface to complete the cyclotron motion. This Fermi-arc mechanism of the 3D quantum Hall effect is characterized by the one-sided edge states, which reside at one side on the top surface but the opposite side on the bottom. The quantized Hall conductance has been observed in the topological Dirac semimetal $\mathrm{Cd}_{3} \mathrm{As}_{2}{ }^{34-37}$. However, the Dirac semimetal consists of two time-reversed Weyl semimetals; thus, on a single surface, the time-reversed Fermi-arc surface states could also support a conventional 2D quantum Hall effect. To identify the top-bottom Fermi-arc 3D quantum Hall effect, a wedge-shaped Hall bar has been used, in which an extra in-plane magnetic field can induce a geometric phase that depends on the sample thickness ${ }^{37}$. As the measurement position of the Hall voltage electrodes is changed, the effective thickness is changed, and a systematic shift of the quantum Hall conductance plateaus occurs (Fig. 1c), strongly supporting the Fermi-arc origin of this 3D quantum Hall effect.

The quantum Hall effect observed in $\mathrm{ZrTe}_{5}{ }^{38}$ has a different mechanism, owing to the formation of CDW. For 3D materials under magnetic fields, the Fermi energy usually crosses the Landau bands. Thus, the bulk states contribute unquantized Hall conductance. However, forming of CDW states can open a band gap at the Fermi energy (Fig. 1e). In real space, the electrons, that form the CDW states along the magnetic field direction, are distributed periodically with the period $\lambda$ (half of the Fermi wavelength). This makes the material look like a stack of 2D electron layers. Therefore, the quantized Hall resistivity is,

$\rho_{x y}=\frac{h}{e^{2}} \lambda$

In the experiment ${ }^{38}$, there are several evidences to support the CDW mechanism of the 3D quantum Hall effect. First, the 3D ellipsoidal Fermi surface of the samples is confirmed by the Shubnikov-de Haas oscillation measurements. This excludes the possibility that the system is originally composed of 2D electron layers. Second, the Hall resistivity shows plateaus when the magnetic field strength is approximately $2 \mathrm{~T}$, at which time the longitudinal resistivity also drops to zero (Fig. 1d). The value of quantized Hall resistivity is consistent with Eq. (1), and $\lambda$ is found to be approximately half of the Fermi wavelength along the magnetic field direction for all four samples, i.e., $\lambda=\pi / k_{\mathrm{F}, z}$ consistent with the feature of the CDW wavelength. Third, the existence of the CDW is supported by the non-Ohmic I-V characteristic (Fig. 1f). The non-Ohmic I-V characteristic is another

\footnotetext{
'Department of Physics, Harbin Institute of Technology, Harbin 150001, China. ${ }^{2}$ Shenzhen Institute for Quantum Science and Engineering and Department of Physics, Southern University of Science and Technology (SUSTech), Shenzhen 518055, China. ${ }^{3}$ Shenzhen Key Laboratory of Quantum Science and Engineering, Shenzhen 518055, China. ${ }^{4}$ Department of Physics, Shanghai Normal University, Shanghai 200234, China. International Center for Quantum Materials, School of Physics, Peking University, Beijing 100871, China. ${ }^{6}$ Beijing Academy of Quantum Information Sciences, Beijing 100193, China. ${ }^{7}$ CAS Center for Excellence in Topological Quantum Computation, University of Chinese Academy of Sciences, Beijing 100190, China. ${ }^{凶}$ email: luhz@sustech.edu.cn
} 
a
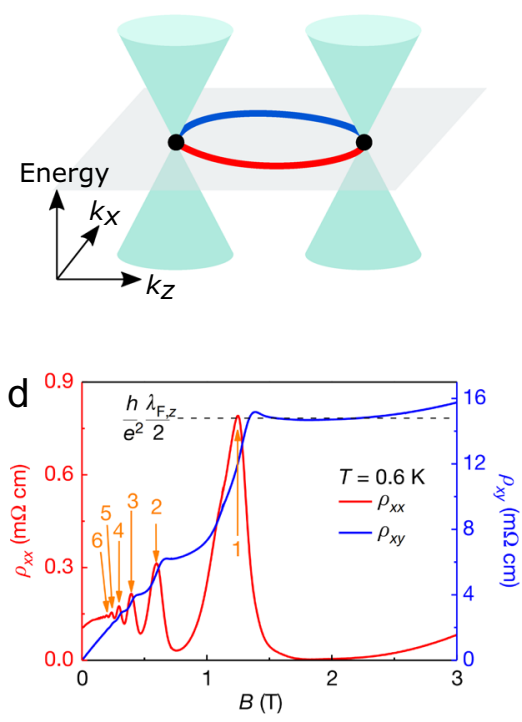

b

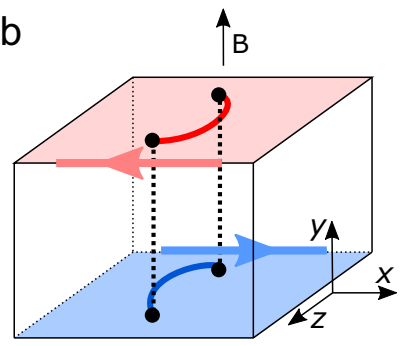

$\mathrm{e}$

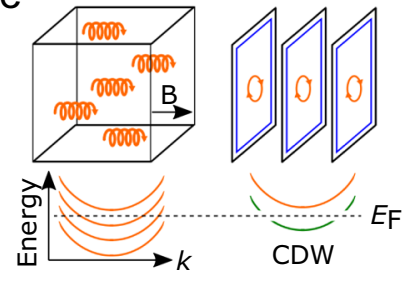

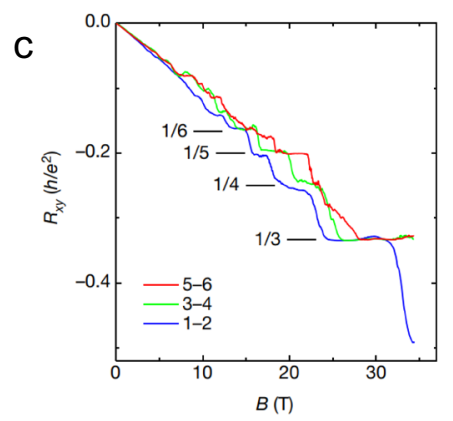

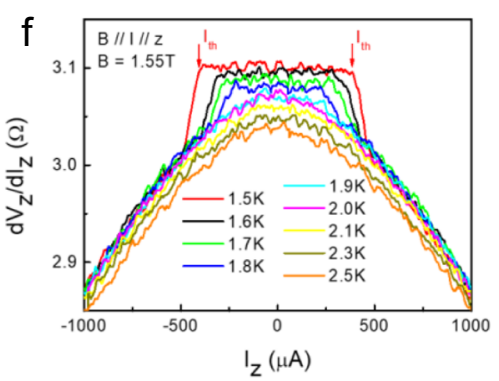

Fig. 1 3D quantum Hall effect. a The energy dispersion of the 3D bulk states (green) of a Weyl semimetal, and the Fermi arcs (the Fermi surface of the topologically protected surface states, red for the top surface and blue for the bottom surface). The black spots denote the Weyl nodes. b Schematic of the Fermi-arc mechanism of the 3D quantum Hall effect in Weyl semimetals. The red and blue arrows depict the hinge states. c The Hall resistance measured at three pairs of Hall electrodes (of different cross-sectional thicknesses) in a wedge-shaped $\mathrm{Cd}_{3} \mathrm{As}_{2}$ sample. Adapted from Ref. ${ }^{37}$. d The Hall $\left(\rho_{x y}\right)$ and longitudinal $\left(\rho_{x x}\right)$ resistivities measured for the charge-density-wave mechanism of the 3D quantum Hall effect in $\mathrm{ZrTe}_{5}$. e Left: Fermi energy crossing the Landau bands in 3D materials; right: bulk gap in Landau bands induced by charge-density wave (CDW). $\mathbf{f}$ Non-Ohmic I-V characteristic in $\mathrm{ZrTe}_{5} . \mathbf{d}$ and $\mathbf{f}$ are adapted from Ref. ${ }^{38}$.

important signal of CDW, because along the CDW direction, the bias voltage or current has to overcome the order parameter (i.e., the gap induced by the CDW). Theoretical calculations reveal the underlying physics in more detail ${ }^{42,43}$. The calculations show that electron-phonon interactions, rather than electron-electron interactions, induce the CDW. They also show that there is a crossover from commensurate (i.e., the CDW wavelength is an integral multiple of the lattice constant) to incommensurate CDW, as a function of the magnetic field. The plateau near $2 \mathrm{~T}$ is due to the commensurate CDW. Later, another experiment has confirmed the Hall resistivity plateau in $\mathrm{ZrTe}_{5}{ }^{44}$, but the plateau was accompanied by a residual longitudinal resistivity. The Hall resistivity plateau accompanied by a residual longitudinal resistivity has also been observed in the compound $\mathrm{HfTe}_{5}{ }^{30,45}$. The significance of the CDW mechanism is that the magnetic field can simultaneously induce two different kinds of phase transition, i.e., a topological phase transition in the plane perpendicular to the field and an order-parameter phase transition in the direction parallel to the field.

Quantized Hall conductance in nodal-line semimetals has also been theoretical predicted ${ }^{46-48}$ although no corresponding experiments have been reported yet. In Ref. ${ }^{47}$, the flat Landau bands at zero energy with large degeneracy occur when the magnetic field is perpendicular or parallel to the nodal ring. This is possibly caused by the chosen low energy model that hosts the strict flat surface states. The authors suggest that the flat Landau bands at zero energy can contribute quantized Hall conductance. Since the results are mainly based on the analysis of energy dispersion, further explicit calculations on transport are expected. Another research ${ }^{48}$ found that a surface quantum Hall conductance can exist in a specific energy window in nodal-line semimetals. The quantum Hall effect was calculated numerically; in addition, a wedge-shaped device was proposed to distinguish the surface and bulk quantum Hall effects.

\section{NONLINEAR HALL EFFECT}

The nonlinear Hall effect refers to the second-order (or quadratic) transverse voltage (or current) in response to a longitudinal current (or electric field). As shown in Fig. 2(a), when an excitation current $I_{\omega}$ with a low frequency $\omega$ is applied, a double frequency transverse voltage $V_{2 \omega}$ can be measured, and a quadratic currentvoltage dependence $V_{2 \omega} \propto\left(I_{\omega}\right)^{2}$ is exhibited. Different from all of the previous Hall effects measured in the linear-response regime, the nonlinear Hall effect does not require time-reversal symmetry breaking by an external magnetic field or magnetism but requires inversion symmetry breaking ${ }^{49,50}$.

Theoretically, the nonlinear Hall effect is formulated as a transverse current induced by a longitudinal electric field. In a two-dimensional system, when an electric field $E_{x}$ is applied along the $x$ direction, the induced nonlinear Hall current can be described by $\xi_{y x x} E_{x} E_{x}+X_{y x x} E_{x} E_{x} e^{i 2 \omega t}$. Besides the double frequency signal, there is also a direct current. It is well known that the linear Hall effect can be described by an integral of the Berry curvature $^{5,51}$. By contrast, the intrinsic nonlinear Hall conductivity $X_{y x x}$ is proportional to the Berry curvature dipole

$D_{x z}=\int \frac{d^{2} k}{(2 \pi)^{2}} \frac{\partial \Omega_{k}^{z}}{\partial k_{x}} f_{0}$,

where $\Omega_{k}^{z}$ is the Berry curvature, and $f_{0}$ is the Fermi distribution function. The above equation can be rewritten as $D_{x z}=$ $-\int \frac{d^{2} k}{(2 \pi)^{2}} \Omega_{k}^{z} \frac{\partial f_{0}}{\partial k_{x}}$ after the application of the integration by parts. This indicates that at low temperature, the Berry curvature dipole is a property at the Fermi energy. The minimal model to study the theory of the nonlinear Hall effect is a tilted massive Dirac model (Fig. 2b), because it has both abundant Berry curvature near the band edges and required breaking of inversion symmetry ${ }^{50}$. The study ${ }^{52}$ on the lattice models also shows that in Weyl semimetals, the Berry curvature dipole can also be contributed by the electrons in the region where two Weyl cones merge together. 
a
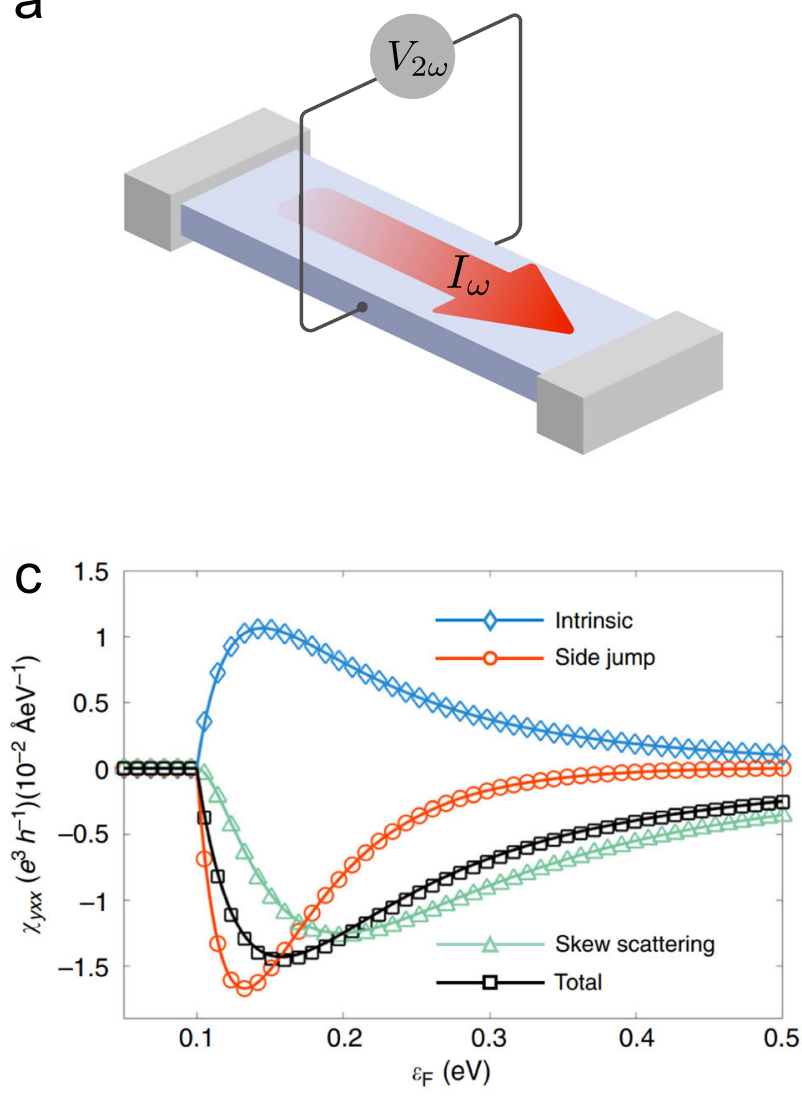

b
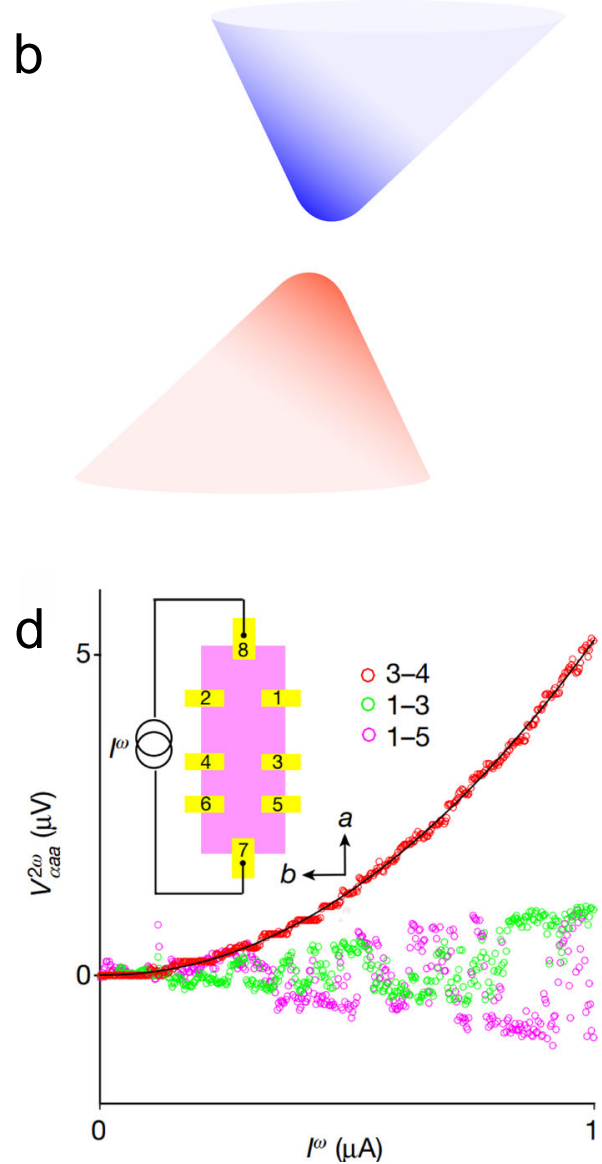

Fig. 2 Nonlinear Hall effect. a Schematic of the measurement setup of the nonlinear Hall effect. b Energy dispersion of a tilted massive 2D Dirac cone, which is the minimal model that supports the nonlinear Hall effect; the blue and red shades represent the Berry curvature with opposite signs. c Different components of the nonlinear Hall conductivity $X_{y x x}$ including the intrinsic, side-jump, and skew-scattering contributions, calculated by the Boltzmann equation. Adapted from Ref. ${ }^{53}$. d The measured nonlinear voltages $V_{a a a}$ along the longitudinal (1-3 and 1-5) and transverse (3-4) directions. The inset is the Hall bar device. Adapted from Ref. ${ }^{60}$.

Furthermore, it has been found that the disorder scattering in the nonlinear Hall effect is equivalently important as the intrinsic contribution ${ }^{53-57}$. The disorder contributions can be classified into the side-jump and skew-scattering mechanisms, similar to those in the anomalous Hall effect ${ }^{2}$. They can even result in a sign change of the nonlinear Hall response (Fig. 2c). In addition, the nonlinear Hall effect can also occur in other mechanisms; for example, the combination of the anomalous velocity and the chiral anomaly in Weyl semimetals can induce the Hall conductivity, which is linear in both electric and magnetic fields ${ }^{58,59}$.

The nonlinear Hall effect has been reported in the bilayer and the few-layer $\mathrm{WTe}_{2}$, respectively ${ }^{60,61}$. The inversion symmetry is broken owing to the $\mathrm{WTe}_{2}$ layer stacking arrangement. Convincingly, the double frequency Hall signal is directly measured by a lock-in amplifier in a phase-sensitive manner, and it is indeed quadratic on the excitation current, as shown in Fig. $2 \mathrm{~d}$. The zerofrequency voltage has also been observed, and it has a similar amplitude to the double frequency voltage ${ }^{61}$. Besides, the nonlinear Hall signal has a $\theta$-dependent on the angle between the excitation current and the principal axis, which is distinguished from the $2 \theta$-dependent linear planar Hall effect ${ }^{61}$. In the experiments ${ }^{60,61}$, the frequency of the driving current is very low $(10-200 \mathrm{~Hz})$, and the experimental results barely depend on the frequency. The nonlinear Hall effect has also been observed in other materials, such as TalrTe ${ }_{4}^{62}, \mathrm{Bi}_{2} \mathrm{Se}_{3}$ films ${ }^{63}, \mathrm{Cd}_{3} \mathrm{As}_{2}{ }^{64}$, monolayer $\mathrm{WSe}_{2}{ }^{65}$, and bilayer graphene ${ }^{66}$.

\section{PERSPECTIVE}

The emergence of the 3D quantum Hall effect and nonlinear Hall effect has brought fresh blood to condensed matter physics, and many research opportunities are offered.

For the Fermi-arc mechanism of the 3D quantum Hall effect, first, many Weyl semimetals have been predicted by first-principle calculations; therefore, more experiments should be conducted on these Weyl semimetal candidates, particularly those with few Weyl points. In addition to $\mathrm{Cd}_{3} \mathrm{As}_{2}$, it is possible to realize the edge states, which only reside on one side of the surface. Second, Fermi arcs are a distinct feature of topological semimetals, it is interesting and meaningful to make extended studies on this Fermi-arc mechanism. For example, studies can be conducted on the systems higher than three dimensions, which can be achieved with the help of synthetic dimensions ${ }^{67}$, and on the higher-order topological semimetals ${ }^{68,69}$, where the hinge states may support 3D quantum Hall effect without applying a magnetic field. For the $3 \mathrm{D}$ quantum Hall effect based on the CDW mechanism, currently, the most important task is to determine the conditions required for the formation of the magnetic-field-induced CDW states. The investigation can start with a systematic study on the temperature and magnetic field dependence of the resistance along the CDW direction. Meanwhile, measurements that directly observe the CDW are expected, such as measurements using the X-ray diffraction or optical methods. The nonlocal measurements are also worth conducting because the vanishing longitudinal 
resistance indicates the dissipationless transport. Another fascinating topic is the $3 D$ version of the fractional Hall effect. The fractional multiplier of $\frac{e^{2}}{h}$ is combined with the period of the CDW state $\lambda$, and $\lambda$ can take different integer multiples of the lattice constant. Therefore, it is important to distinguish the 3D fractional quantum Hall effect and the different commensurate CDW states. Furthermore, one can search the 3D quantum anomalous Hall effect in the context of magnetic materials. Except the CDW mechanism, the gap opening could be induced by other correlation phases, such as the spin-density wave, Winger crystal, or excitonic insulator. They may have different features of the quantized Hall resistivity compared with the CDW case. This also provides another scenario to study the strongly-correlated states in strong magnetic fields.

Although significant progress has been made in the nonlinear Hall effect, there are still many open questions and challenges. From the experimental aspect, despite the challenges in device fabrication, richer phenomena could be observed in three dimensions. The measurement could be generalized to using two perpendicular excitation currents with different frequencies, allowing more symmetry properties to be probed. From the theoretical aspect, although the nonlinear Hall effect has been intensively studied based on the semi-classical Boltzmann transport theory, a consistent quantum description is still underway $^{70}$, in particular, for better describing the disorder effect. Manipulating the nonlinear Hall signal by controlling disorder configurations might be possible in the future. In addition, the temperature effect is worth addressing in theoretical studies because the nonlinear Hall effect observed in experiments normally persists to a pretty high temperature. There are also several straighfarward directions, including finding more materials that host significant nonlinear Hall effects, for example, among non-centrosymmetric materials and Dirac materials; investigating the details of the nonlinear Hall effect in specific band structures or materials; and examining the nonlinear response tensors according to the theoretical symmetry analysis.

The exploration of the new members of the Hall family is still in its early stage. More exciting discoveries are expected in the future.

Received: 26 May 2021; Accepted: 21 October 2021;

Published online: 25 November 2021

\section{REFERENCES}

1. Hall, E. On a new action of the magnet on electric currents. Am. J. Math. 2, 287-292 (1879).

2. Nagaosa, N., Sinova, J., Onoda, S., MacDonald, A. H. \& Ong, N. P. Anomalous Hall effect. Rev. Mod. Phys. 82, 1539-1592 (2010).

3. Sinova, J., Valenzuela, S. O., Wunderlich, J., Back, C. H. \& Jungwirth, T. Spin Hall effects. Rev. Mod. Phys. 87, 1213-1260 (2015).

4. Klitzing, K. V., Dorda, G. \& Pepper, M. New method for high-accuracy determination of the fine-structure constant based on quantized Hall resistance. Phys. Rev. Lett. 45, 494-497 (1980)

5. Thouless, D. J., Kohmoto, M., Nightingale, M. P. \& den Nijs, M. Quantized Hall conductance in a two-dimensional periodic potential. Phys. Rev. Lett. 49, 405-408 (1982).

6. Tsui, D. C., Stormer, H. L. \& Gossard, A. C. Two-dimensional magnetotransport in the extreme quantum limit. Phys. Rev. Lett. 48, 1559-1562 (1982).

7. Laughlin, R. B. Anomalous quantum Hall effect: an incompressible quantum fluid with fractionally charged excitations. Phys. Rev. Lett. 50, 1395-1398 (1983).

8. Bernevig, B. A., Hughes, T. L. \& Zhang, S.-C. Quantum spin Hall effect and topological phase transition in HgTe quantum wells. Science 314, 1757-1761 (2006).

9. König, M. et al. Quantum spin Hall insulator state in HgTe quantum wells. Science 318, 766-770 (2007).

10. Hasan, M. Z. \& Kane, C. L. Colloquium: topological insulators. Rev. Mod. Phys. 82, 3045-3067 (2010).

11. Qi, X.-L. \& Zhang, S.-C. Topological insulators and superconductors. Rev. Mod. Phys. 83, 1057-1110 (2011).
12. $\mathrm{Yu}, \mathrm{R}$. et al. Quantized anomalous Hall effect in magnetic topological insulators. Science 329, 61-64 (2010).

13. Chang, C.-Z. et al. Experimental observation of the quantum anomalous Hall effect in a magnetic topological insulator. Science 340, 167-170 (2013).

14. Armitage, N. P., Mele, E. J. \& Vishwanath, A. Weyl and Dirac semimetals in threedimensional solids. Rev. Mod. Phys. 90, 015001 (2018).

15. Ozawa, T. et al. Topological photonics. Rev. Mod. Phys. 91, 015006 (2019).

16. Fang, C., Weng, H., Dai, X. \& Fang, Z. Topological nodal line semimetals. Chin. Phys. B 25, 117106 (2016).

17. Zhang, X., Xiao, M., Cheng, Y., Lu, M.-H. \& Christensen, J. Topological sound. Commun. Phys. 1, 97 (2018).

18. Halperin, B. I. Possible states for a three-dimensional electron gas in a strong magnetic field. Jpn. J. Appl. Phys. 26, 1913 (1987).

19. Montambaux, G. \& Kohmoto, M. Quantized Hall effect in three dimensions. Phys. Rev. B 41, 11417-11421 (1990).

20. Kohmoto, M., Halperin, B. I. \& Wu, Y.-S. Diophantine equation for the threedimensional quantum Hall effect. Phys. Rev. B 45, 13488-13493 (1992).

21. Koshino, M., Aoki, H., Kuroki, K., Kagoshima, S. \& Osada, T. Hofstadter butterfly and integer quantum Hall effect in three dimensions. Phys. Rev. Lett. 86, 1062-1065 (2001).

22. Bernevig, B. A., Hughes, T. L., Raghu, S. \& Arovas, D. P. Theory of the threedimensional quantum Hall effect in graphite. Phys. Rev. Lett. 99, 146804 (2007).

23. Störmer, H. L., Eisenstein, J. P., Gossard, A. C., Wiegmann, W. \& Baldwin, K. Quantization of the Hall effect in an anisotropic three-dimensional electronic system. Phys. Rev. Lett. 56, 85-88 (1986).

24. Cooper, J. R. et al. Quantized Hall effect and a new field-induced phase transition in the organic superconductor (TMTSF) ${ }_{2} \mathrm{PF}_{6}$. Phys. Rev. Lett. 63, 1984-1987 (1989).

25. Hannahs, S. T., Brooks, J. S., Kang, W., Chiang, L. Y. \& Chaikin, P. M. Quantum Hall effect in a bulk crystal. Phys. Rev. Lett. 63, 1988-1991 (1989).

26. Hill, S. et al. Bulk quantum Hall effect in $\eta-\mathrm{Mo}_{4} \mathrm{O}_{11}$. Phys. Rev. $B$ 58, 10778-10783 (1998).

27. Liu, J. Y. et al. Spin-valley locking and bulk quantum Hall effect in a noncentrosymmetric Dirac semimetal BaMnSb. Nat. Commun. 12, 4062 (2021).

28. Li, H., Liu, H., Jiang, H. \& Xie, X. C. 3D quantum Hall effect and a global picture of edge states in Weyl semimetals. Phys. Rev. Lett. 125, 036602 (2020).

29. Cheng, S.-G., Jiang, H., Sun, Q.-F. \& Xie, X. C. Quantum Hall effect in wedge-shaped samples. Phys. Rev. B 102, 075304 (2020).

30. Wang, P. et al. Approaching three-dimensional quantum hall effect in bulk $\mathrm{HfTe}_{5}$. Phys. Rev. B 101, 161201(R) (2020).

31. Chang, M., Geng, H., Sheng, L. \& Xing, D. Y. Three-dimensional quantum Hall effect in Weyl semimetals. Phys. Rev. B 103, 245434 (2021).

32. Wang, C. M., Sun, H.-P., Lu, H.-Z. \& Xie, X. C. 3D quantum Hall effect of Fermi arcs in topological semimetals. Phys. Rev. Lett. 119, 136806 (2017).

33. Lu, H.-Z. Perspective: 3D quantum Hall effect. Natl Sci. Rev. 6, 208 (2019).

34. Zhang, C. et al. Room-temperature chiral charge pumping in Dirac semimetals. Nat. Commun. 8, 13741 (2017).

35. Uchida, M. et al. Quantum Hall states observed in thin films of Dirac semimetal $\mathrm{Cd}_{3} \mathrm{As}_{2}$. Nat. Commun. 8, 2274 (2017).

36. Schumann, T. et al. Observation of the quantum Hall effect in confined films of the three-dimensional Dirac semimetal $\mathrm{Cd}_{3} \mathrm{As}_{2}$. Phys. Rev. Lett. 120, 016801 (2018).

37. Zhang, $C$. et al. Quantum Hall effect based on Weyl orbit in $\mathrm{Cd}_{3} A s_{2}$. Nature 565, 331-336 (2019).

38. Tang, F. et al. Three-dimensional quantum Hall effect and metal-insulator transition in $\mathrm{ZrTe}_{5}$. Nature 569, 537-541 (2019).

39. Potter, A. C., Kimchi, I. \& Vishwanath, A. Quantum oscillations from surface Fermi arcs in Weyl and Dirac semimetals. Nat. Commun. 5, 5161 (2014).

40. Moll, P. J. W. et al. Transport evidence for Fermi-arc-mediated chirality transfer in the Dirac semimetal $\mathrm{Cd}_{3} \mathrm{As}_{2}$. Nature 535, 266-270 (2016).

41. Zhang, Y., Bulmash, D., Hosur, P., Potter, A. C. \& Vishwanath, A. Quantum oscillations from generic surface Fermi arcs and bulk chiral modes in Weyl semimetals. Sci. Rep. 6, 23741 (2016).

42. Qin, F. et al. Theory for the charge-density-wave mechanism of 3D quantum Hall effect. Phys. Rev. Lett. 125, 206601 (2020).

43. Zhao, P.-L., Lu, H.-Z. \& Xie, X. C. Theory for magnetic-field-driven 3D metalinsulator transitions in the quantum limit. Phys. Rev. Lett. 127, 046602 (2021).

44. Galeski, S. et al. Origin of the quasi-quantized Hall effect in $\mathrm{ZrTe}_{5}$. Nat. Commun. 12, 3197 (2021).

45. Galeski, S. et al. Unconventional Hall response in the quantum limit of $\mathrm{HfTe}_{5}$. Nat. Commun. 11, 5926 (2020)

46. Mullen, K., Uchoa, B. \& Glatzhofer, D. T. Line of Dirac nodes in hyperhoneycomb lattices. Phys. Rev. Lett. 115, 026403 (2015).

47. Molina, R. A. \& González, J. Surface and 3D quantum Hall effects from engineering of exceptional points in nodal-line semimetals. Phys. Rev. Lett. 120, 146601 (2018). 
48. Zhao, G.-Q., Rui, W. B., Wang, C. M., Lu, H.-Z. \& Xie, X. C. Quantized Hall conductance in 3D topological nodal-line semimetals without chiral symmetry. Preprint at https://arxiv.org/abs/2004.01386 (2020)

49. Sodemann, I. \& Fu, L. Quantum nonlinear Hall effect induced by Berry curvature dipole in time-reversal invariant materials. Phys. Rev. Lett. 115, 216806 (2015).

50. Du, Z. Z., Wang, C. M., Lu, H.-Z. \& Xie, X. C. Band signatures for strong nonlinear Hall effect in bilayer WTe $e_{2}$. Phys. Rev. Lett. 121, 266601 (2018).

51. Xiao, D., Chang, M. C. \& Niu, Q. Berry phase effects on electronic properties. Rev. Mod. Phys. 82, 1959-2007 (2010).

52. Zeng, C., Nandy, S. \& Tewari, S. Nonlinear transport in Weyl semimetals induced by Berry curvature dipole. Phys. Rev. B 103, 245119 (2021).

53. Du, Z. Z., Wang, C. M., Li, S., Lu, H.-Z. \& Xie, X. C. Disorder-induced nonlinear Hall effect with time-reversal symmetry. Nat. Commun. 10, 3047 (2019).

54. Isobe, H., Xu, S.-Y. \& Fu, L. High-frequency rectification via chiral Bloch electrons. Sci. Adv. 6, eaay2497 (2020)

55. Nandy, S. \& Sodemann, I. Symmetry and quantum kinetics of the nonlinear Hall effect. Phys. Rev. B 100, 195117 (2019).

56. König, E. J., Dzero, M., Levchenko, A. \& Pesin, D. A. Gyrotropic Hall effect in Berrycurved materials. Phys. Rev. B 99, 155404 (2019).

57. Xiao, C., Du, Z. Z. \& Niu, Q. Theory of nonlinear Hall effects: Modified semiclassics from quantum kinetics. Phys. Rev. B 100, 165422 (2019).

58. Li, R.-H., Heinonen, O. G., Burkov, A. A. \& Zhang, S. S.-L. Nonlinear Hall effect in Weyl semimetals induced by chiral anomaly. Phys. Rev. B 103, 045105 (2021).

59. Nandy, S., Zeng, C. \& Tewari, S. Chiral anomaly induced nonlinear Hall effect in multi-Weyl semimetals. Preprint at https://arxiv.org/abs/2104.14969 (2021).

60. $\mathrm{Ma}, \mathrm{Q}$. et al. Observation of the nonlinear Hall effect under time-reversalsymmetric conditions. Nature 565, 337-342 (2019).

61. Kang, K., Li, T., Sohn, E., Shan, J. \& Mak, K. F. Observation of the nonlinear anomalous Hall effect in 2D WTe 2 . Nat. Mater. 18, 324-328 (2019).

62. Kumar, D. et al. Room-temperature nonlinear Hall effect and wireless radiofrequency rectification in Weyl semimetal TalrTe 4 . Nat. Nanotechnol. 16, 421-425 (2021).

63. He, P. et al. Quantum frequency doubling in the topological insulator $\mathrm{Bi}_{2} \mathrm{Se}_{3} . \mathrm{Nat}$. Commun. 12, 1-7 (2021).

64. Shvetsov, O. O., Esin, V. D., Timonina, A. V., Kolesnikov, N. N. \& Deviatov, E. V. Nonlinear Hall effect in three-dimensional Weyl and Dirac semimetals. JETP Lett. 109, 715-721 (2019).

65. Qin, M.-S. et al. Strain tunable Berry curvature dipole, orbital magnetization and nonlinear Hall effect in WSe 2 monolayer. Chin. Phys. Lett. 38, 017301 (2021).

66. Ho, S.-C. et al. Hall effects in artificially corrugated bilayer graphene without breaking time-reversal symmetry. Nat. Electron. 4, 116-125 (2021).

67. Ozawa, T. \& Price, H. M. Topological quantum matter in synthetic dimensions. Nat. Rev. Phys. 1, 349-357 (2019).

68. Xie, B. et al. Higher-order band topology. Nat. Rev. Phys. 3, 520-532 (2021).

69. Chen, R., Liu, T., Wang, C. M., Lu, H.-Z. \& Xie, X. C. Field-tunable one-sided higher-order topological hinge states in Dirac semimetals. Phys. Rev. Lett. 127, 066801 (2021).

70. Du, Z. Z., Wang, C. M., Sun, H.-P., Lu, H.-Z. \& Xie, X. C. Quantum theory of the nonlinear Hall effect. Nat. Commun. 12, 5038 (2021).

\section{ACKNOWLEDGEMENTS}

This work was supported by the National Natural Science Foundation of China (11534001, 11974249, 11925402, 12004157, 11404106), the National Basic Research Program of China (2015CB921102), the Strategic Priority Research Program of Chinese Academy of Sciences (XDB28000000), Guangdong province (2016ZT06D348, 2020KCXTD001), the Natural Science Foundation of Shanghai (19ZR1437300), Shenzhen High-level Special Fund (G02206304, G02206404), and the Science, Technology and Innovation Commission of Shenzhen Municipality (ZDSYS20170303165926217, JCYJ20170412152620376, KYTDPT20181011104202253), the China Postdoctoral Science Foundation (2019M662150, 2020T130635), SUSTech Presidential Postdoctoral Fellowship, and Center for Computational Science and Engineering of Southern University of Science and Technology.

\section{AUTHOR CONTRIBUTIONS}

S.L. wrote the manuscript with the assistance from H.-Z.L., C.M.W., Z.Z.D., F.Q. and X.C.X

\section{COMPETING INTERESTS}

The authors declare no competing interests.

\section{ADDITIONAL INFORMATION}

Correspondence and requests for materials should be addressed to Hai-Zhou Lu.

Reprints and permission information is available at http://www.nature.com/ reprints

Publisher's note Springer Nature remains neutral with regard to jurisdictional claims in published maps and institutional affiliations.

\begin{abstract}
Open Access This article is licensed under a Creative Commons Attribution 4.0 International License, which permits use, sharing, adaptation, distribution and reproduction in any medium or format, as long as you give appropriate credit to the original author(s) and the source, provide a link to the Creative Commons license, and indicate if changes were made. The images or other third party material in this article are included in the article's Creative Commons license, unless indicated otherwise in a credit line to the material. If material is not included in the article's Creative Commons license and your intended use is not permitted by statutory regulation or exceeds the permitted use, you will need to obtain permission directly from the copyright holder. To view a copy of this license, visit http://creativecommons. org/licenses/by/4.0/.
\end{abstract}

(c) The Author(s) 2021 